Provided for non-commercial research and education use. Not for reproduction, distribution or commercial use.

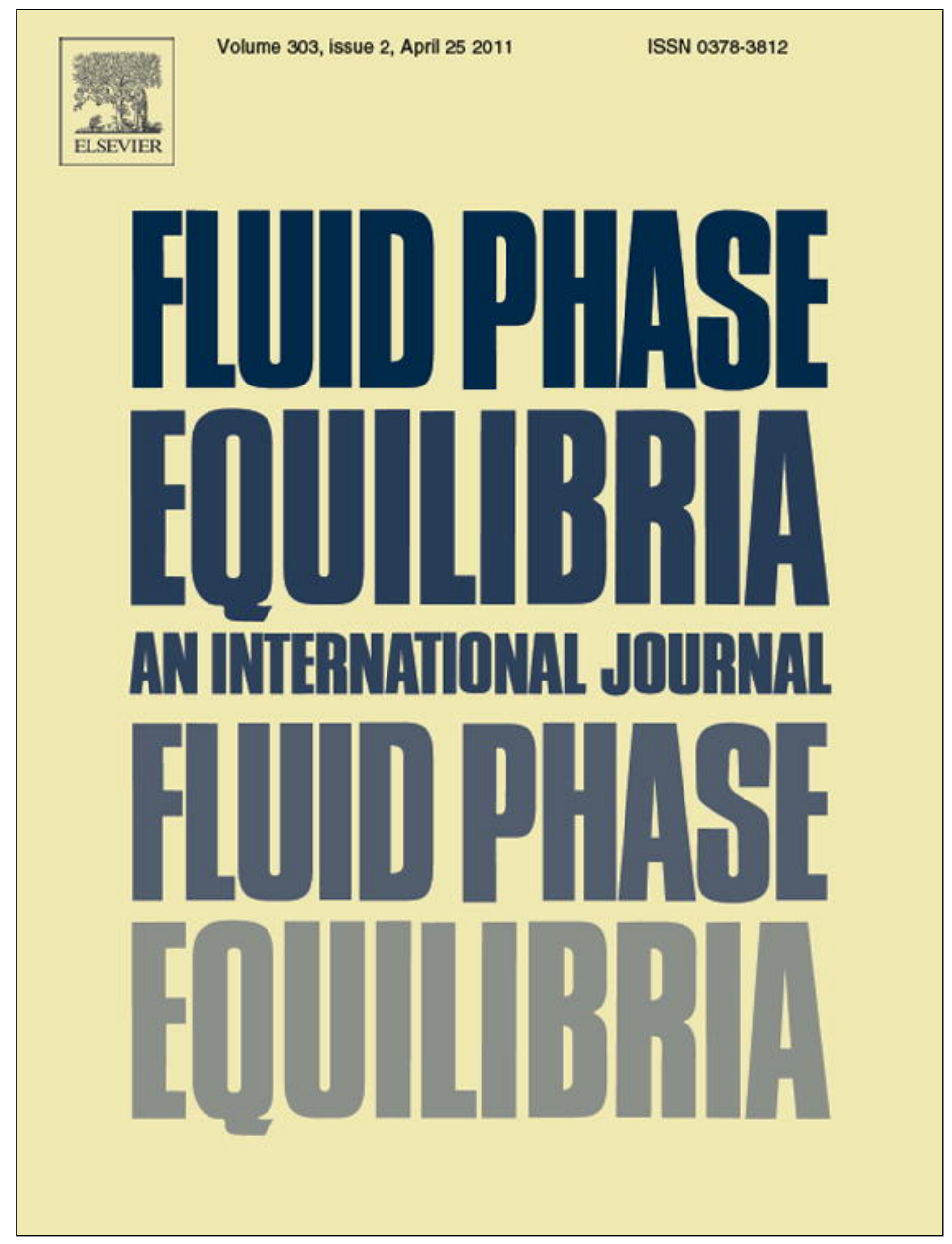

This article appeared in a journal published by Elsevier. The attached copy is furnished to the author for internal non-commercial research and education use, including for instruction at the authors institution and sharing with colleagues.

Other uses, including reproduction and distribution, or selling or licensing copies, or posting to personal, institutional or third party websites are prohibited.

In most cases authors are permitted to post their version of the article (e.g. in Word or Tex form) to their personal website or institutional repository. Authors requiring further information regarding Elsevier's archiving and manuscript policies are encouraged to visit:

http://www.elsevier.com/copyright 


\title{
On the behaviour of solutions of xenon in liquid cycloalkanes: Solubility of xenon in cyclopentane
}

\author{
Rui P.M.F. Bonifácio a ${ }^{\text {, Eduardo J.M. Filipe }}{ }^{\mathrm{a}, *}$, M. Carolina dos Ramos ${ }^{\mathrm{b}, \mathrm{c}}$, \\ Felipe J. Blas ${ }^{c}$, Luís F.G. Martins ${ }^{\mathrm{d}}$ \\ a Centro de Química Estrutural, Instituto Superior Técnico, 1049-001 Lisboa, Portugal \\ ${ }^{\mathrm{b}}$ Department of Chemical and Biomolecular Engineering, Vanderbilt University, Nashville, TN 37235, USA \\ c Departamento de Física Aplicada, Facultad de Ciencias Experimentales, Universidad de Huelva, 21071 Huelva, Spain \\ d Centro de Química de Évora, Universidade de Évora, Rua Romão Ramalho 59, 7000-671 Évora, Portugal
}

\section{A R T I C L E I N F O}

Article history:

Received 4 August 2010

Received in revised form 19 October 2010

Accepted 1 February 2011

\section{Keywords:}

Gas solubility

Xenon

Cycloalkanes

\begin{abstract}
A B S T R A C T
The solubility of xenon in liquid cyclopentane has been studied experimentally and theoretically. Measurements of the solubility of xenon in liquid cyclopentane are reported as a function of temperature from $254.60 \mathrm{~K}$ to $313.66 \mathrm{~K}$. The imprecision of the experimental data is less than $0.3 \%$. The thermodynamic functions of solvation of xenon in cyclopentane, such as the standard Gibbs energy, enthalpy, entropy and heat capacity of solvation, have been calculated from the temperature dependence of Henry's law coefficients. The results provide further information about the differences between the xenon + cycloalkanes and the xenon $+n$-alkane interactions. In particular, interaction enthalpies between xenon and $\mathrm{CH}_{2}$ groups in $n$ alkanes and cycloalkanes have been estimated and compared. Using a version of the soft-SAFT approach developed to model cyclic molecules, we were able to reproduce the experimental solubility for xenon in cyclopentane using simple Lorentz-Berthelot rules to describe the unlike interaction.
\end{abstract}

(c) 2011 Elsevier B.V. All rights reserved.

\section{Introduction}

Cyclic molecular structures are very common in nature. Consequently, the study of their thermodynamic behaviour, in particular cycloalkanes, has always been a matter of great interest and an attractive research field from both experimental and theoretical points of view [1]. However, the number of theoretical approaches, which can provide a reliable and accurate description of the thermodynamic behaviour of cyclic molecules and their mixtures, is rather small, compared with those dealing with linear molecules. $n$-Alkanes and cycloalkanes, though essentially formed by the same type of chemical units $\left(\mathrm{CH}_{2}\right.$ groups), display rather different physical behaviour either as pure substances or in mixtures. Many studies have compared the thermodynamic behaviour exhibited by cycloalkanes with that of their corresponding $n$-alkanes, i.e., linear molecules with the same number of carbon atoms. In general, it is found that cycloalkanes are less volatile, denser and have higher vapourization enthalpies than their linear analogues. This has been

\footnotetext{
* Corresponding author at: Departamento de Engenharia Quimica, Instituto Superior Técnico, Av. Rovisco Pais, 1049-001 Lisboa, Portugal. Tel.: +351 218419 261; fax: +351218464 455 .

E-mail address: efilipe@ist.utl.pt (E.J.M. Filipe).
}

interpreted as a result of stronger attractive interactions between cyclic molecules, ultimately due to their different molecular structure (electronic structure and shape) and organization in the liquid state [1].

Cycloalkanes can be divided into three categories: (1) long chain cycloalkanes, with more than 9 carbon atoms. These adopt molecular conformations that basically resemble double chain linear alkanes; (2) medium chain cycloalkanes, 6-9 carbon atoms. These molecules, of which cyclohexane is by far the most studied one, are quasi-spherical globular molecules. They can act as 'structure breakers' when mixed with long $n$-alkanes, i.e., they disrupt the short-range orientational order of $n$-alkanes, giving rise to positive contributions to the excess enthalpies and entropies; (3) short cycloalkanes, with 5 carbon atoms or less. These are rigid, plate like anisotropic rotors. Rotations around $\mathrm{C}-\mathrm{C}$ bonds are hindered, resulting in almost eclipsed conformations of the $\mathrm{CH}_{2}$ groups. The distortion of the bond angles causes a large strain and displaces the electronic density out of the ring. These important differences at the molecular level could be expected to influence the intermolecular interactions between $\mathrm{CH}_{2}$ groups of small cycloalkanes [1].

Liquid state properties are known to depend considerably on the organization of the fluid, for which molecular shape (repulsion forces) is a key factor. With that in mind, we have undertaken a number of studies with the purpose of assessing the 
effect of the molecular shape on the thermodynamic behaviour of liquid mixtures, in particular those involving cyclic molecules $[2,3]$. From the experimental point of view, binary mixtures such as xenon + cyclopropane [4] and xenon + cyclobutane [5] have been investigated and the results compared with those of mixtures containing the linear analogues xenon + propane [6] and xenon + butane [7]. In general, we have found that mixtures containing a cyclic component exhibit larger positive deviations from Raoult's Law (and consequently larger values of $\mathrm{G}^{\mathrm{E}}$ ), but more negative excess volumes and enthalpies than those containing a linear analogue. These results have been explained using the so-called "condensation" effect proposed in the 1970s by Patterson et al. [8]. According to this effect, linear alkanes would "condense" at the surface of highly branched, rigid and sterically hindered molecules, with a restriction of its molecular motion and hence giving rise to negative contributions to excess enthalpies and entropies and positive additions to excess heat capacities. This effect was also used to explain the results exhibited by mixtures involving cyclopentane $[9,10]$, whose plate-like anisotropic molecular structure would favour the condensation of $n$-alkanes on its surface. Since cyclopropane and cyclobutane are also plate-like anisotropic rotors, it was suggested that a "condensation" type phenomenon could arise between these molecules and xenon, a particle with a high polarizability, which enhances the dispersion forces [4,5].

In order to accurately predict the thermodynamic properties of complex systems from a theoretical point of view, it is essential to incorporate explicitly the molecular features of a given system. There are in the literature a number of approaches to predict the thermodynamic properties of complex substances and their mixtures. In this work we focus on the use of a molecular-based formalism, the so-called soft-SAFT equation of state proposed by Blas and Vega [11,12]. We suggest the book of Sengers et al. [13] for readers interested on the details of this and other molecular theories.

The Statistical Associating Fluid Theory (SAFT) is a molecularbased equation of state, originally proposed by Chapman et al. $[14,15]$ based on Wertheim's first-order Thermodynamic Perturbation Theory [16-21], specially suited to predict the thermodynamic properties of chain molecules and associating substances. In this approach, the different microscopic effects are accounted for in several contributions to the Helmholtz free energy. In particular, the theory explicitly takes into account the molecular non-sphericity of chain molecules, flexibility, and specific interactions (hydrogen bonding), among others. Since its development, the SAFT approach has been used to predict the phase equilibria of a wide variety of pure components and their mixtures, and it is presently considered one of the most powerful predictive tools for the study of the fluid phase equilibria. For a more detailed description, we recommend the reviews of Müller and Gubbins [22] and Radosz et al. [23]. More recently, there have been new developments related with the formalism of SAFT that are not included in the previous reviews [24-26].

In this work we have extended the study of systems involving xenon and cyclic molecules to consider xenon + cyclopentane solutions. The solubility of xenon in liquid cyclopentane has been measured as a function of temperature from $254 \mathrm{~K}$ to $314 \mathrm{~K}$ and Henry's law coefficients calculated. This system had been previously studied by Pollack et al. [27] in the range 278.15-303.15 K. The present work not only doubles the temperature range of the available results, but also provides experimental data with a precision that allows the calculation of the thermodynamic properties of solvation as a function of temperature and therefore the calculation of second derivative properties, such as the heat capacity of solvation.

The results were interpreted using an extension of the soft-SAFT equation of state that deals with ring molecular structures $[3,28]$.
For comparison, we have also calculated the solubility of xenon in $n$-pentane. This procedure allows examining the effect of changing a linear alkane by a cyclic alkane on the thermodynamic properties of a given mixture.

\section{Experimental}

The cyclopentane used as solvent was from Acros, analytical reagent, with $99 \%$ ( $\mathrm{mol} / \mathrm{mol}$ ) minimum stated purity. The liquid was purified by distillation in an inert atmosphere of dry nitrogen. The final purity was confirmed by checking its vapour pressure, after degasification, at two temperatures. Deviations from literature values [29] were found to be $0.39 \%$ at $258.33 \mathrm{~K}$ and $-0.21 \%$ at $277.17 \mathrm{~K}$. The xenon used was from Linde Gas with $99.99 \%$ $(\mathrm{mol} / \mathrm{mol})$ minimum stated purity. The gas was used as received from the manufacturer.

The experimental apparatus and procedure have been previously described in detail [30]. The solubility measurements involve the equilibration of known amounts of dry gas and degassed solvent at constant volume and the determination of the equilibrium pressure for the saturated solution maintained at a constant temperature.

The amount of gas is determined measuring its pressure in a calibrated glass bulb at constant temperature and correcting for gas non-ideality. Pressure is measured with a transducer (Paroscientific model $0-7$ bar, precision $0.01 \%$ FS). The pure solvent is degassed by successive melting/freezing cycles, while vacuum pumping noncondensable gases. The amount of pure solvent is determined volumetrically. The equilibrium cell, based on the design of Carnicer [31], has two capillaries through which the liquid is forced to circulate during the equilibration process, promoting a close contact with the gas. The volume of the cell was previously determined with a precision of $0.01 \%$. The readings of pressure during the dissolution process are recorded until a constant value is reached indicating that equilibrium has been attained. The final pressure, temperature and level of the solution in the capillaries are then measured. Equilibrium is typically attained within $72 \mathrm{~h}$.

Temperature is maintained constant in a water thermostat to within $0.01 \mathrm{~K}$ by means of a Hart Scientific PID temperature controller, and is measured with a previously calibrated Pt100 platinum resistance thermometer. The measurement of solubility at different temperatures is done by simply changing the thermostat set point and waiting for a new thermodynamic equilibrium. With a single loading it is thus possible to make measurements over a large temperature range. Several runs were performed in order to check the reproducibility of the results, both increasing and decreasing temperature.

\section{Experimental results}

The solubility of xenon in cyclopentane was experimentally measured from $254.60 \mathrm{~K}$ to $313.66 \mathrm{~K}$. The results are reported in Table 1. For each experimental point, the temperature, equilibrium pressure, molar fractions of xenon in the liquid and gaseous phases in equilibrium and Henry's law coefficients, $H_{2,1}\left(T, p_{1}^{\text {sat }}\right)$, are indicated.

Henry's law coefficients were calculated from experimental data as follows. Henry's law coefficients are usually defined as [32],

$H_{2,1}(T, p)=\lim _{x_{2} \rightarrow 0}\left[\frac{f_{2}\left(p, T, x_{2}\right)}{x_{2}}\right]$

where $f_{2}$ is the fugacity of the solute (component 2) and $x_{2}$ its molar fraction in the liquid solution. The fugacity of component 2 can then be determined in the usual way [33]:

$f_{2}\left(p, T, x_{2}\right)=\varphi_{2}(p, T) y_{2} p$ 
Table 1

Values for the Henry's law coefficients, equilibrium pressure, $p$, and xenon molar fraction in the gaseous and liquid phases at equilibrium, between 254 and $313 \mathrm{~K}$, for solutions of xenon in cyclopentane.

\begin{tabular}{lclll}
\hline$T / \mathrm{K}$ & $p / \mathrm{kPa}$ & $x_{2}\left(10^{-2}\right)$ & $y_{2}$ & $H_{2,1}\left(p_{1}^{\text {sat }}, T\right) / \mathrm{MPa}$ \\
\hline 254.60 & 84.69 & 3.478 & 0.9390 & 2.267 \\
258.69 & 54.49 & 1.919 & 0.8789 & 2.483 \\
265.64 & 60.73 & 1.797 & 0.8424 & 2.832 \\
269.43 & 64.55 & 1.733 & 0.8201 & 3.038 \\
277.18 & 90.43 & 2.129 & 0.8141 & 3.432 \\
286.23 & 104.1 & 1.959 & 0.7568 & 3.990 \\
286.23 & 85.70 & 1.491 & 0.7033 & 4.017 \\
295.21 & 120.5 & 1.814 & 0.6939 & 4.571 \\
304.46 & 141.2 & 1.687 & 0.6251 & 5.185 \\
313.66 & 166.6 & 1.580 & 0.5560 & 5.807 \\
\hline
\end{tabular}

with

$\varphi_{2}(p, T)=\exp \left[\frac{p\left(B_{22}+y_{1}^{2} \delta_{12}\right)}{R T}\right]$

where $\varphi_{2}$ is the fugacity coefficient of component 2, $p$ is the vapour pressure of the saturated solution and $y_{2}$ the solute mole fraction in the vapour phase. $B_{22}$ is the second virial coefficient of the solute and $\delta_{12}$ is given by $=2 B_{12}-B_{11}-B_{22}$, where $B_{11}$ is the second virial coefficient of the solvent and $B_{12}$ is the solute-solvent crossed second virial coefficient.

The equilibrium compositions were calculated iteratively from experimentally measured quantities allowing for non-ideality of the gaseous mixture. The process starts with initial guesses for the molar fractions in both phases and continues with the determination of the quantities of solute and solvent present in the two phases. The process converges rapidly and coherent values for $y_{i}$ and $x_{i}$ are obtained after a few iterations. The calculation of Henry's law coefficients is then immediate, using Eqs. (1)-(3). For comparison purposes it is useful to express Henry's coefficients at the same pressure, namely the vapour pressure of the pure solvent. The pressure dependence for $H_{2,1}$ can be expressed as [34]

$H_{2,1}(T, p)=H_{2,1}\left(T, p_{1}^{\text {sat }}\right) \exp \int_{p_{1}^{\text {sat }}}^{p}\left\lfloor\frac{V_{2}^{\infty}(p, T)}{R T} d p\right\rfloor$

where $V_{2}^{\infty}$ is the partial molar volume of the solute at infinite dilution and $p_{1}^{\text {sat }}$ is the vapour pressure of the pure solvent.

The vapour pressure of cyclopentane was taken from Ref. [35] and the molar volume calculated from Refs. [36-39]. The second virial coefficients for pure cyclopentane were taken from Dymond et al. [40] and for pure xenon from the compilation of Dymond and Smith [41]. The crossed virial coefficient for the mixture was estimated using the Tsonopoulos correlation [35]. As a first approximation, the partial molar volumes of xenon in cyclopentane, necessary for the calculation of the Henry's law coefficients (Eq. (4)), were estimated using the method described by Tiepel and Gubbins $[42,43]$. This method has been applied to calculate the partial molar volume of several gases in water and proved to be accurate to within $3 \%$. This uncertainty affects negligibly the values of the Henry's law constant.

Henry's law coefficients, $H_{2,1}\left(T, p_{1}^{\text {sat }}\right)$, are plotted as a function of temperature in Fig. 1. The solubility of xenon in cyclopentane has

Table 2

Coefficients for Eq. (5), correlating the experimental data for xenon + cyclopentane solutions.

\begin{tabular}{ll}
\hline Coefficients of Eq. (5) & Xenon + cyclopentane \\
\hline$A_{0}$ & +24.903 \\
$A_{1}$ & -19.977 \\
$A_{2}$ & -2.588 \\
AAD & $+0.3 \%$
\end{tabular}

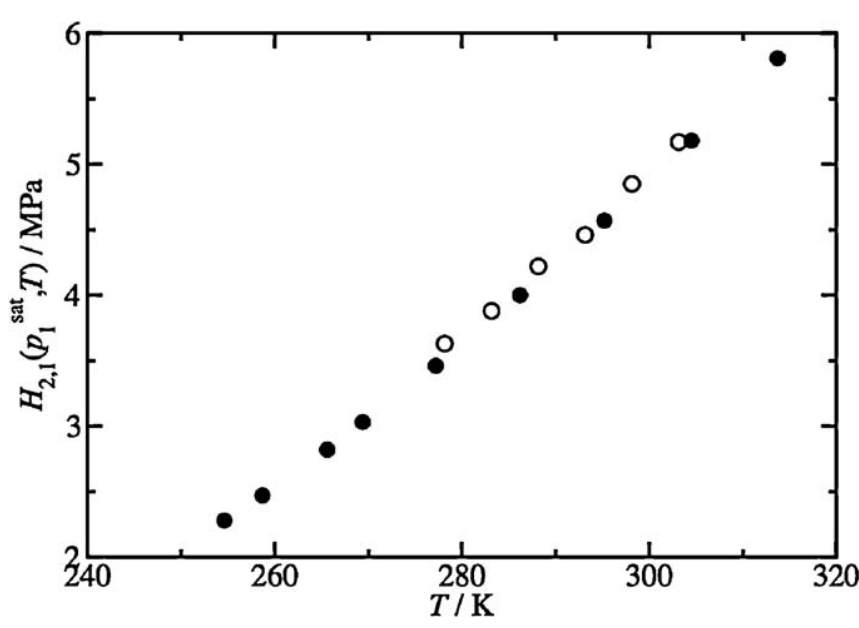

Fig. 1. Henry's law coefficients for (xenon + cyclopentane): $(\bullet)$ this work; $(\bigcirc)$ Pollack et al. [27].

been previously measured by Pollack et al. [27] at six temperatures. Their values show positive deviations from ours up to $3.5 \%$ and a dispersion of $3 \%$, which were included in Fig. 1 for comparison.

The dependence of the Henry's law coefficient in relatively narrow temperature ranges can be represented by the equation proposed by Clarke and Glew [44]:

$$
\begin{aligned}
\ln H_{2,1}\left(T, p_{1}^{\text {sat }}\right)= & A_{0}+\frac{A_{1}}{10^{-2} T / \mathrm{K}}+A_{2} \ln \left(10^{-2} T / \mathrm{K}\right)+A_{3}\left(10^{-2} T / \mathrm{K}\right) \\
& +A_{4}\left(10^{-2} T / \mathrm{K}\right)^{2}+\cdots
\end{aligned}
$$

The experimental results were fitted to Eq. (5) and the coefficients $A_{i}$ are listed in Table 2 . The relative deviations of the experimental results from the smoothing correlation do not exceed $\pm 0.5 \%$ and the percentage of average absolute deviation (AAD) is $0.3 \%$.

The change in molar Gibbs energy when the solute is transferred, at temperature $T$, from the pure perfect gas state at standard pressure, to the dilute state in the solvent (standard Gibbs energy of solvation) [45] is given by [34],

$\Delta G_{2}^{0}\left(T, p_{1}^{\text {sat }}\right)=R T \ln \left[\frac{H_{2,1}\left(T, p_{1}^{\text {sat }}\right)}{p^{0}}\right]$

where $p^{0}$ is the standard state pressure, considered as $101,325 \mathrm{~Pa}$. The standard enthalpy and entropy of solvation can be obtained by calculating the corresponding partial derivatives of the Gibbs energy with respect to temperature at constant pressure. The result for the enthalpy of solvation at temperature $T$ and at the vapour pressure of pure solvent is:

$$
\begin{aligned}
\Delta H_{2}^{0}\left(T, p_{1}^{s a t}\right)= & -R T^{2}\left[\frac{d}{d T}\left(\frac{\ln H_{2,1}\left(T, p_{1}^{s a t}\right)}{p^{0}}\right)\right. \\
& \left.-\frac{V_{2}^{\infty}(T)}{R T}\left(\frac{d p_{1}^{s a t}(T)}{d T}\right)\right]
\end{aligned}
$$

while the standard entropy of solvation is given by,

$$
\begin{aligned}
\Delta S_{2}^{0}\left(T, p_{1}^{\text {sat }}\right)= & \frac{R \ln H_{2,1}\left(T, p_{1}^{\text {sat }}\right)}{p^{0}}-R T \frac{d}{d T}\left(\frac{\ln H_{2,1}\left(T, p_{1}^{\text {sat }}\right)}{p^{0}}\right) \\
& -V_{2}^{\infty}\left(\frac{d p_{1}^{\text {sat }}(T)}{d T}\right)
\end{aligned}
$$


Table 3

Thermodynamic functions of solvation for xenon in cyclopentane. $\Delta G_{2}^{0}$ is the molar Gibbs energy of solvation, $\Delta H_{2}^{0}$ is the molar enthalpy of solvation and $\Delta S_{2}^{0}$ is the molar entropy of solvation. The values are based on the ideal gas state at 101,325 Pa.

\begin{tabular}{lcll}
\hline$T / \mathrm{K}$ & $\Delta G_{2}^{0} / \mathrm{kJ} \mathrm{mol}^{-1}$ & $\Delta H_{2}^{0} / \mathrm{kJ} \mathrm{mol}^{-1}$ & $\Delta S_{2}^{0} / \mathrm{J} \mathrm{K}^{-1} \mathrm{~mol}^{-1}$ \\
\hline 254.6 & 6.587 & -11.13 & -69.6 \\
258.7 & 6.872 & -11.04 & -69.2 \\
265.6 & 7.348 & -10.89 & -68.7 \\
269.4 & 7.608 & -10.81 & -68.3 \\
277.2 & 8.139 & -10.63 & -67.7 \\
286.2 & 8.746 & -10.44 & -67.0 \\
295.2 & 9.347 & -10.24 & -66.3 \\
304.5 & 9.961 & -10.03 & -65.6 \\
313.7 & 10.56 & -9.818 & -65.0 \\
\hline
\end{tabular}

The thermodynamic functions of solvation were calculated from the Clarke and Glew smoothing equation, for (xenon + cyclopentane) solutions. The results are listed in Table 3. No values obtained directly by calorimetric determinations could be found for comparison.

\section{Molecular model and theory}

The soft-SAFT approach is a modified version of the SAFT by Blas and Vega $[11,12]$ to describe associating and non-associating chains. The reference system is the Lennard-Jones fluid, instead of the hard-sphere fluid, which allows simplifying the theory since the dispersive and repulsive terms are taken into account simultaneously. In addition, the use of a soft potential also allows the association sites to be fully embedded inside the reference core, and thus some overlapping could occur between segments with bonded associating sites, which is a more realistic situation.

Homonuclear linear chain and ring-like molecules are then described using the united-atom model approach. Linear chains and rings are formed by $m$ Lennard-Jones segments tangentially bonded. Each segment is characterized by a diameter or segment size $\left(\sigma_{i i}\right)$, and a dispersive energy $\left(\varepsilon_{i i}\right)$. The inter- and intra-molecular interactions between the segments are taken into account through the Lennard-Jones potential. These models had been used previously in the literature to accurately represent a great variety of molecules, including xenon $[3,6,7,46-48]$ and $n-$ alkanes [12,49-53]. They have also been used to predict the phase behaviour of pure and binary systems, including cyclic molecules [3,28] and hypothetical cycloalkane molecules [5].

The soft-SAFT theory, as other versions of SAFT, is written in terms of the Helmholtz free energy, which can be expressed as a sum of different microscopic effects: ideal contribution term $\left(A^{I D E A L}\right)$ for the Helmholtz free energy of an ideal mixture of chains; the reference Lennard-Jones contribution term $\left(A^{L J}\right)$ to account for the repulsions and attractions between the segments that forms the molecules; the chain or ring contribution term $\left(A^{\text {CHAIN/RING }}\right)$ accounting for the formation of the chain [54,55] or ring [28] molecules, and the association contribution term $\left(A^{A S S O C}\right)$ which takes into account the contribution due to the hydrogen bonding association. The Helmholtz free energy may by written as [11],

$\frac{A}{N k_{B} T}=\frac{A^{I D E A L}}{N k_{B} T}+\frac{A^{L J}}{N k_{B} T}+\frac{A^{C H A I N / R I N G}}{N k_{B} T}+\frac{A^{A S S O C}}{N k_{B} T}$

where $N$ is the number of molecules, $k_{B}$ is the Boltzmann constant, and $T$ is the temperature. The main features of each individual contribution to the Helmholtz free energy, is explained separately here. In the present work, we shall not consider the association term since we are dealing with non-associating systems.

\subsection{Ideal term}

The Helmholtz free energy of an ideal mixture of $n$ chains or ring-like molecules can be written in the following way,

$\frac{A^{I D E A L}}{N k_{B} T}=\sum_{i=1}^{n} x_{i} \ln \left(\rho_{i} \Lambda_{i}^{3}\right)-1$

where $x_{i}=N_{i} / N$ is the molar fraction, $\rho_{i}=N_{i} / V$ is the molecular density, $\Lambda_{i}$ is the thermal de Broglie wavelength, $N_{i}$ is the number of molecules of component $i$, and $V$ the volume. The segment density, $\rho_{s}$, is easily related to the total molecular density, $\rho$, through,

$\rho_{s}=\sum_{i=1}^{n} m_{i} \rho_{i}=\left(\sum_{i=1}^{n} m_{i} x_{i}\right) \rho$

with $m_{i}$ the molecular length (or number of segments per molecule) of species $i$.

\subsection{Lennard-Jones reference term}

The Lennard-Jones reference term accounts for both the repulsive and attractive interactions between the segments forming the molecules. Although the Lennard-Jones potential is relatively simple, it cannot be solved theoretically to obtain an analytical expression of the Helmholtz free energy. In this work we use the Lennard-Jones equation of state proposed by Johnson et al. [56]. This equation is an extended Benedict-Webb-Rubin equation of state that was fitted to simulation data for pure Lennard-Jones fluids over a broad range of temperatures and densities.

\subsection{Chain/ring term}

The contribution to the chain/ring formation is accounted for in $A^{\text {CHAIN/RING }}$. The chain contribution term was independently introduced by Wertheim [20], and Jackson et al. [54,55] from the first-order perturbation theory for associating spherical molecules [16-19]. The Helmholtz free energy due to the formation of a mixture of Lennard-Jones chains with bond lengths equal to $\sigma_{i i}$, the diameter of the Lennard-Jones segments in species $i$, takes the form of,

$\frac{A^{\text {CHAIN }}}{N k_{B} T}=-\sum_{i=1}^{n} x_{i}\left(m_{i}-1\right) \ln y_{i i}^{L J}\left(\sigma_{i i}\right)$

while the free energy due to the formation of a mixture of LennardJones ring-like molecules with lengths equal to $\sigma_{j j}$, the diameter of the Lennard-Jones segments in species $j$, is given by [28],

$\frac{A^{R I N G}}{N k_{B} T}=-\sum_{i=1}^{n} x_{i}\left(m_{i}\right) \ln y_{i i}^{L J}\left(\sigma_{i i}\right)$

In both cases, Eqs. (12) and (13), $y_{i i}^{L J}\left(\sigma_{i i}\right)$ is the contact value of the cavity correlation function of spherical segments of species $i$ in the Lennard-Jones reference fluid mixture. $y_{i i}^{L J}\left(\sigma_{i i}\right)$ is easily related to the pair radial distribution function of the Lennard-Jones fluid, $g_{i i}^{L J}\left(\sigma_{i i}\right)$. Note that the difference between these two equations is that in the chain contribution term only $\left(m_{i}-1\right)$ contacts per molecule are counted, while the ring contribution term takes into account one extra contact, e.g., $m_{i}$ contacts are counted. Obviously, the free energy due to the formation of a mixture of chain and ring molecules is given by the adequate combination of Eqs. (12) and (13). 


\subsection{Extension to mixtures}

Since the Helmholtz free energy is calculated by adding different terms, each of them should be expressed in terms of compositions for mixtures studied. In the soft-SAFT version, only the reference term needs to be extended to treat mixtures [11]. The ideal and chain/ring contributions depend explicitly on composition and thus, are readily applicable to mixtures. We use the van der Waals one-fluid theory (vdW-1f) to describe the $A^{L J}$ term of the mixture [57]. In this theory, the residual Helmholtz free energy of the mixture is approximated by the residual Helmholtz free energy of a pure hypothetical fluid, with parameters $\sigma_{m}$ and $\varepsilon_{m}$, calculated from,

$\sigma_{m}^{3}=\frac{\sum_{i=1}^{n} \sum_{j=1}^{n} m_{i} m_{j} x_{i} x_{j} \sigma_{i j}^{3}}{\sum_{i=1}^{n} \sum_{j=1}^{n} m_{i} m_{j} x_{i} x_{j}}$

$\varepsilon_{m} \sigma_{m}^{3}=\frac{\sum_{i=1}^{n} \sum_{j=1}^{n} m_{i} m_{j} x_{i} x_{j} \varepsilon_{i j} \sigma_{i j}^{3}}{\sum_{i=1}^{n} \sum_{j=1}^{n} m_{i} m_{j} x_{i} x_{j}}$

where the mixing rules for a Lennard-Jones mixture have been expressed as functions of the molecular molar fractions $x_{i}$. To obtain the pair radial distribution function of a mixture of Lennard-Jones spheres, the same mixing rules (vdW-1f) [57] have been used, as in previous works [11,12]. Results from Johnson et al. [56] are used for the pair radial distribution function of the Lennard-Jones fluid from extensive computer simulations fitted to an empirical function of the reduced temperature and density. Details of the simulations are reported elsewhere [56].

For the unlike interaction parameters, we employ the LorentzBerthelot combining rules [57], which are expressed as follows:

$\sigma_{i j}=\frac{\sigma_{i i}+\sigma_{j j}}{2}$

$\varepsilon_{i j}=\left(\varepsilon_{i i} \varepsilon_{j j}\right)^{1 / 2}$

\subsection{Molecular parameters}

The soft-SAFT formalism needs three molecular parameters, $m$, the chain length, $\varepsilon / k_{B}$, the monomeric dispersive energy, and $\sigma$, the segment size of the monomers, $i$ to describe the thermodynamic properties of pure substances. Pámies et al. [50] obtained a set of transferable parameters for the $n$-alkane series. This set is able to provide an excellent description of the phase behaviour of linear alkanes, from methane up to long-chain $n$-alkanes. Here we use the Pàmies and Vega parametrization to model the phase behaviour of linear pentane molecule. In principle, one could expect that cycloalkanes would require a new set of molecular parameters, determined by fitting to their vapour pressure and orthobaric liquid density. However, we have found that the same molecular parameters valid for the linear alkanes, when used within the softSAFT formalism specific for ring molecules, provide a fairly good description of the phase behaviour of cycloalkanes. In particular, this choice is able to predict the vapour pressure of cycloalkanes almost quantitatively and the liquid density within $10 \%$ of the experimental result, without any further fitting procedure. The vapour pressure and coexistence density curve of cyclopentane is shown in Fig. 2. It is important to remark that the molecular parameters used for a cycloalkane, with a given number of carbon atoms, are the same as those for its linear analogue, i.e., the $n$-alkane, with the same number of carbon atoms. With this procedure we are, thus, able to quantify the effect of using the ring term (Eq. (13)), instead of the chain term (Eq. (12)) and compare with experimental data.

The molecular parameters of xenon have been obtained following a similar procedure by fitting the saturated liquid density
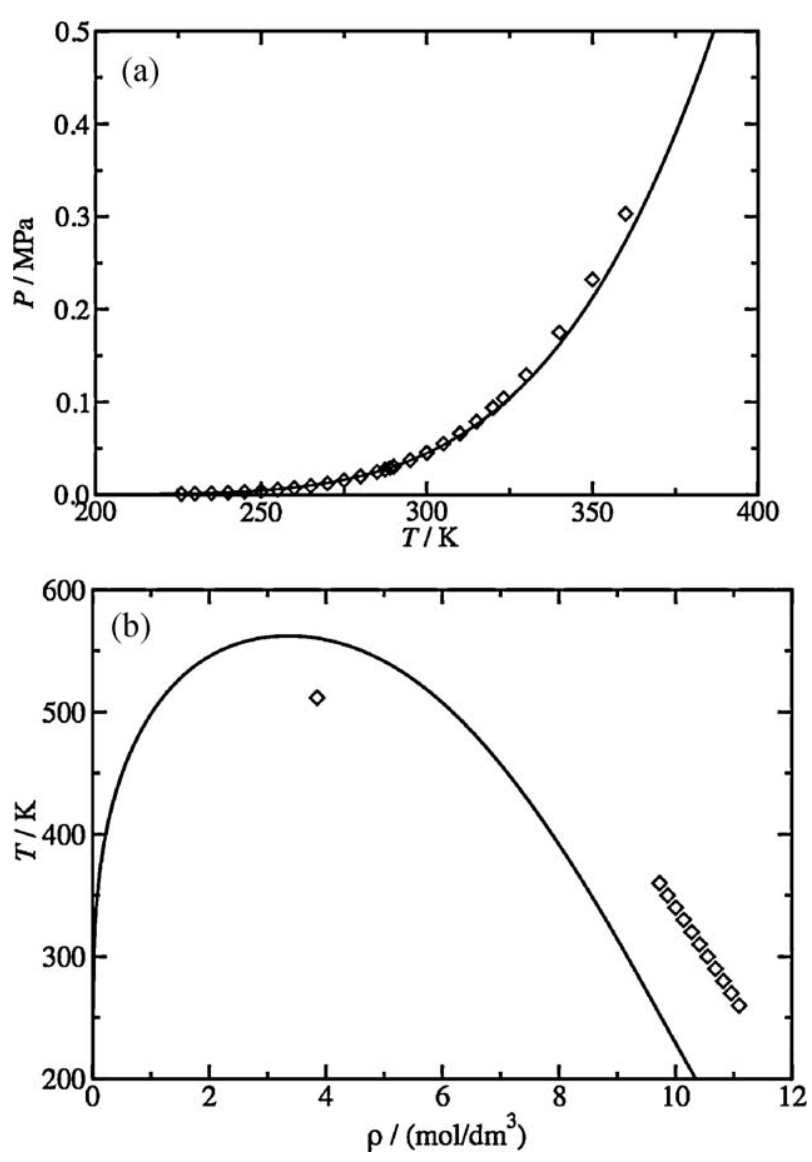

Fig. 2. Vapour pressure (a) and saturated densities (b) as a function of temperature for cyclopentane. Symbols correspond to experimental data $[36,67,68]$, while solid lines represent the theoretical predictions obtained using the soft-SAFT approach.

and equating the chemical potential in both phases. The values of all the molecular parameters used in this work are compiled in Table 4.

\section{Discussion}

Henry's constants of xenon in cyclopentane as a function of temperature, were obtained from the experimental solubility data, using the procedure explained in Section 3 and theoretically, using the soft-SAFT formalism presented in the previous section. Fig. 3 shows the comparison between the experimental data and the theoretical predictions. The theoretical Henry's constants were calculated from the chemical potential of xenon in the solvent at infinite dilution [58]. As can be seen, the soft-SAFT predictions slightly underestimate the experimental data, but the overall agreement can be considered very good. It should be emphasized that: (1) The calculations for xenon + cyclopentane were performed using molecular parameters for $n$-pentane. (2) The calculations were performed without allowing for deviations from the LorentzBerthelot combining rules, i.e., the theoretical results are true

Table 4

Optimized Lennard-Jones intermolecular potential parameters for xenon, $n$-pentane and cyclopentane. ${ }^{\mathrm{a}}$

\begin{tabular}{llll}
\hline Substance & $m$ & $\left(\varepsilon / k_{B}\right) / \mathrm{K}$ & $\sigma / \mathrm{nm}$ \\
\hline Xenon & 1.00 & 226.3 & 0.3959 \\
$n$-Pentane or cyclopentane & 2.497 & 246.6 & 0.3901 \\
\hline
\end{tabular}

a $m$ is the number of spherical segments in the model, $\left(\varepsilon / k_{B}\right)$ the dispersive energy, and $\sigma$ the diameter or size of each segment. 


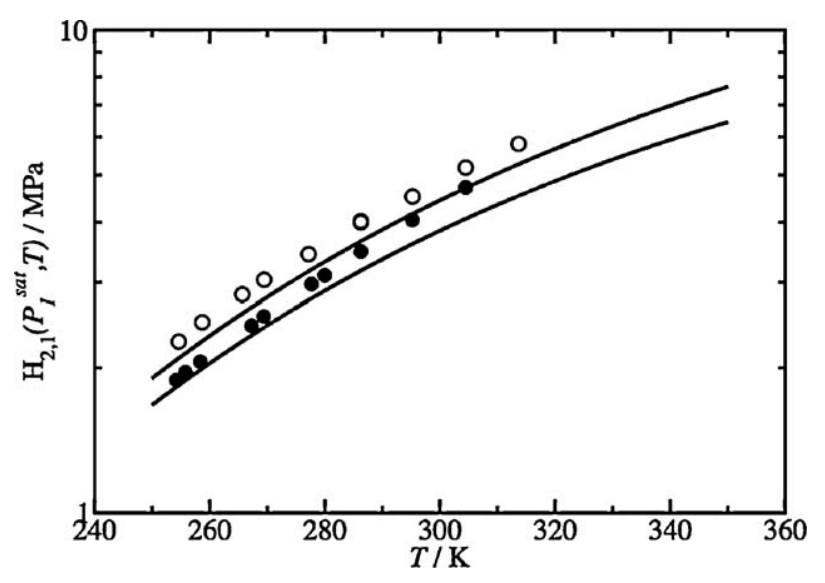

Fig. 3. Henry's law coefficients for: $(\bigcirc)(x e n o n+$ cyclopentane); $(\bullet)(x e n o n+n-$ pentane) [59]; solid lines represent the soft-SAFT predictions.

predictions, since no parameters were fitted to experimental data of the mixtures. In the same figure we have included the experimental and theoretical Henry's constants for the xenon $+n$-pentane system, for comparison. The agreement between the experimental and theoretical results is slightly better in this case, especially in the low temperature range. Nevertheless it is clear that the theory is able to capture the difference of the solubility of xenon in the two solvents. We believe that, in the case of xenon + cyclopentane, the agreement between theory and experiment could be improved if effective parameters were used for cyclopentane. The adopted procedure, however, has the advantage of directly showing the performance of the ring term (Eq. (13)).

The standard enthalpy and entropy of solvation for (xenon+cyclopentane) solutions, as a function of temperature, are shown in Fig. 4. The same properties are also displayed for (xenon $+n$-pentane), for comparison, taken from Ref. [59]. As can be seen, for xenon + cyclopentane, both the enthalpy and entropy of solvation increase monotonously as the temperature is raised. This behaviour opposes that found for solutions of xenon in $n$-pentane and also in $n$-hexane, as recently reported [59]. In that case, the enthalpy of solvation slightly increases at lower temperatures, goes through a maximum and then decreases at higher temperatures. The temperature at which the solvation enthalpy reaches a maximum changes with the molecular weight of the $n$-alkanes $(254.5 \mathrm{~K}$ for xenon $+n$-pentane and $275 \mathrm{~K}$ for xenon $+n$-hexane). Pollack et al. [27] noticed that the average value of the entropy

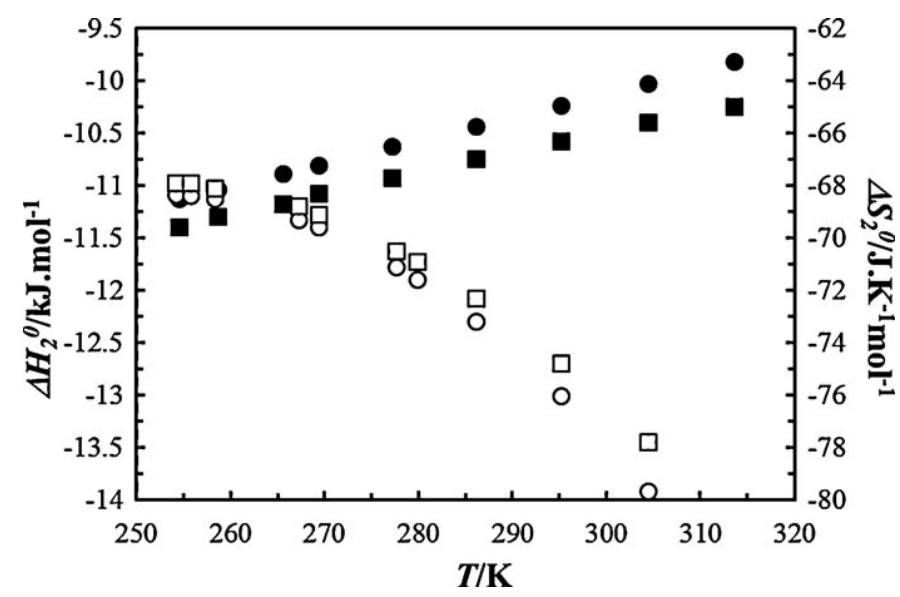
Fig. 4. Enthalpies of solvation, $\Delta H_{2}^{0}$ (circles) and entropies of solvation, $\Delta S_{2}^{0}$
(squares) for (xenon + cyclopentane) (filled symbols, • and $\mathbf{a})$ and (xenon + pentane) [59] (open symbols, $\bigcirc$ and $\square$ ) as a function of temperature. of solvation (determined over the temperature range studied by this author) is not very sensitive to the solvent and was found to be practically constant for solutions of xenon in 45 solvents of different chemical natures. Differences in the enthalpy of solvation from solvent to solvent are in fact more apparent. However, the opposite temperature dependence for both enthalpy and entropy of solvation, now reported for a linear and a cyclic system, could not be observed with the data of Pollack et al.

As previously mentioned, we have found that mixtures of xenon containing a cyclic component (i.e., xenon + cyclopropane [4] and xenon + cyclobutane [5]) exhibit large positive deviations from Raoult's Law (and consequently larger values of $\mathrm{G}^{\mathrm{E}}$ ), but more negative excess volumes and enthalpies than those containing a linear analogue (xenon + propane [6] and xenon + butane [7]).

As recalled above, a "condensation effect" was proposed by Patterson and co-workers to explain the abnormally low values of the heat of mixing for mixtures involving long $n$-alkanes with some sterically hindered branched alkanes [60]. The "condensation" of flexible $n$-alkanes (such as $n$-hexadecane or $n$-octane) at the surface of "rocky" molecules would be the reason for negative contributions to the enthalpy and entropy of mixing. According to Patterson et al. the relative sterical hindrance of each branched alkane molecule was assumed to be inversely proportional to the pure liquid molar volume of each alkane.

By analogy, the condensation effect was later used to explain the unusual behaviour of mixtures involving branched alkanes (e.g. isomers of hexane) and cycloalkanes [61]. The enthalpy of mixing of a branched alkane with a series of different cycloalkanes changes regularly when plotted as a function of the cycloalkane chain length, except for cyclopentane, for which a much lower heat of mixing was obtained. The absence of energetically accessible conformations, which makes cyclopentane a plate-like anisotropic molecule, was the main explanation to this behaviour. The "condensation effect" also influenced excess heat capacity measurements. In mixtures whose behaviour can be explained by "condensation", $C_{P}^{E}$ tends to be positive, whereas negative values of $C_{p}^{E}$ are found for other mixtures [62]. This is consistent with the logical prediction that "condensation" would be less effective with the increasing temperature, leading to lower negative contributions to heat of mixing at higher temperatures.

The accuracy of the results now reported, for the solubility for xenon in cyclopentane as a function of temperature, as well as those for xenon in $n$-pentane from a previous work, allows us to estimate the heat capacity of solvation from the temperature dependence of enthalpy of solvation. This was done by differentiation of Eq. (7), for both systems. The results are displayed in Fig. 5 and seem to confirm the existence of "condensation". In fact, the heat capacity of solvation for solutions of xenon in $n$-pentane is negative (decreasing with increasing temperature) whereas for xenon + cyclopentane, it is positive (slightly increasing with temperature).

The dissolution process of a gas in a liquid is frequently described as the combination of two contributions: a first contribution due to the formation of a cavity with the appropriate size and shape to host the solute molecule; and a second contribution due to the interaction between the solute molecules and the solvent molecules within its coordination sphere. Energetically, the cavity formation is often identified with the vapourization enthalpy of the solvent, $\Delta H_{v}$, corrected for the change in size and shape of the cavity. Irrespectively of the model used to calculate the enthalpy of cavity formation, $H_{c a v}$, the solute-solvent enthalpy of interaction, $H_{\text {int }}$, can then be obtained from the solvation enthalpy as

$H_{\text {int }}=\Delta H_{2}^{0}-H_{\text {cav }}$

In this work we have calculated the enthalpy of cavity formation for xenon in cyclopentane following the procedure 


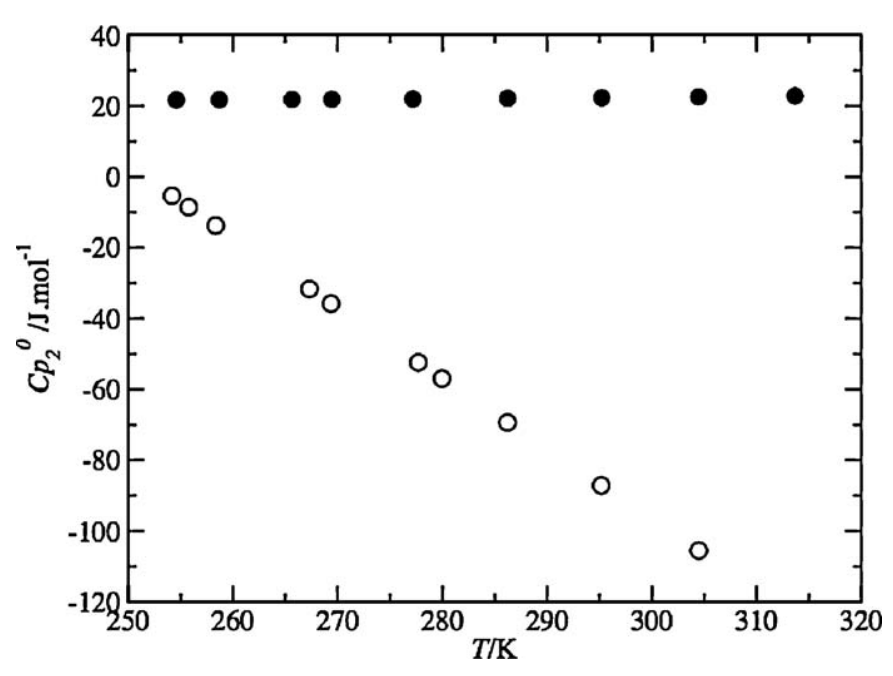

Fig. 5. Heat capacity of solvation $\left(C_{P, 2}^{0}\right)$ for (xenon+cyclopentane) (•) and (xenon + pentane) $[59](\bigcirc)$ as a function of temperature.

proposed by Sinonaglu [63]. This enthalpic contribution can be expressed as

$H_{\text {cav }}=\frac{S_{S}}{S_{S}} \Delta_{v} H$

where $S_{S} / S_{S}$ represent the ratio of the surfaces of the solute and solvent cavities. It can be calculated from partial molar volumes at infinite dilution of the solute in the solvent, molar volumes of the solvent, and simple geometrical considerations.

Fig. 6 shows the calculated interaction enthalpy, as a function of the reduced temperature of the mixture, for the solution of xenon in cyclopentane. The reduced temperature of the mixture, $T_{r}$, is defined here as $T_{r}=T / T_{c_{12}}$ (where $T_{c_{12}}=\left(1-k_{12}\right)\left(T_{c_{1}} T_{c_{2}}\right)^{1 / 2}$, $\left(1-k_{12}\right)=\left(8\left(V_{c_{1}} V_{c_{2}}\right)^{1 / 2}\right) /\left(V_{c_{1}}^{1 / 3}+V_{c_{2}}^{1 / 3}\right)^{3}$ and $T_{c_{1}}, T_{c_{2}}, V_{c_{1}}$ and $V_{c_{2}}$ are, respectively, the critical temperatures and critical volumes of the pure fluids). We have also included the interaction enthalpies for solution of xenon in $n$-pentane and in $n$-hexane. As can be seen, the interaction enthalpy for xenon in cyclopentane is more negative than those corresponding of xenon in $n$-pentane and in $n$-hexane. It is known that xenon- $\mathrm{CH}_{3}$ dispersive interactions are stronger than those corresponding to xenon- $\mathrm{CH}_{2}$ interactions [64]. This explains why the interaction enthalpy of xenon and $n$-pentane is more negative than that of xenon in $n$-hexane, reflecting the higher percentage of $\mathrm{CH}_{3}$ groups in the $n$-pentane molecule. Our

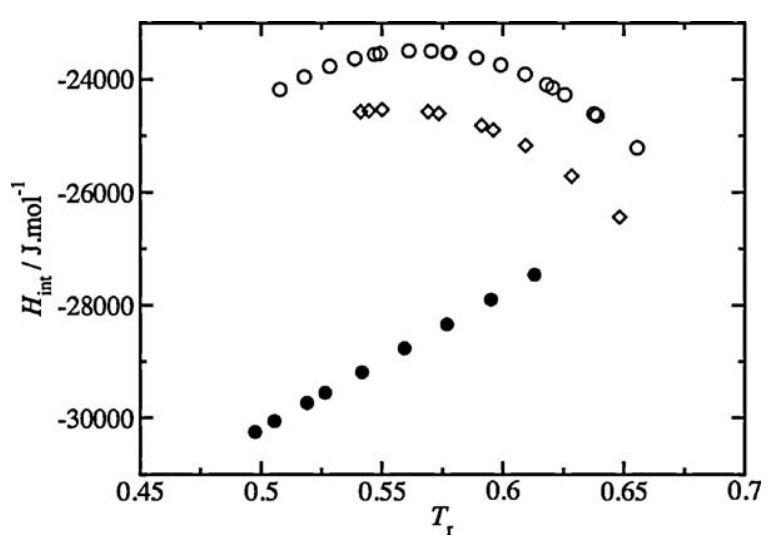

Fig. 6. Interaction enthalpies $\left(H_{\text {int }}\right)$ for $(\bullet)(x e n o n+$ cyclopentane $),(\diamond)(x e n o n+n-$ pentane) and $(\bigcirc)$ (xenon $+n$-hexane) $[59]$ as a function of reduced temperature.

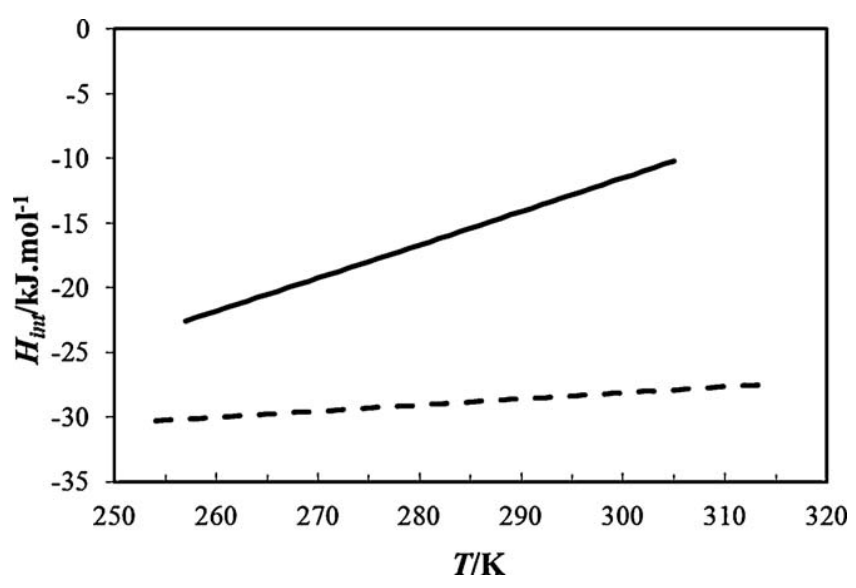

Fig. 7. (Solid line) xenon- $\mathrm{CH}_{2}$ interaction enthalpy contribution, estimated from solutions in linear alkanes ( $n$-pentane and $n$-hexane) [59] and (dashed line) experimental interaction enthalpy for xenon solutions in cyclopentane, as a function of temperature.

results indicate that the interaction enthalpy for solutions of xenon in cyclopentane is more negative than that corresponding to solutions of xenon in both linear alkanes.

Assuming that xenon dissolves in $n$-alkanes at random positions (which implies that the number of $\mathrm{CH}_{2} / \mathrm{CH}_{3}$ groups within xenon's solvation shell depends exclusively on their molecular proportion), the interaction enthalpy for xenon in $n$-pentane and $n$-hexane solutions (Fig. 6) could be separated into its xenon- $\mathrm{CH}_{2}$ and xenon- $\mathrm{CH}_{3}$ contributions. These contributions will be equivalent to interaction enthalpies (per mole of solute) of xenon in hypothetical solvents composed exclusively of $\mathrm{CH}_{3}$ or $\mathrm{CH}_{2}$ units. They should also be almost independent of chain length, which seems a reasonable hypothesis for solutions of xenon in linear alkanes. Moura Ramos and Gonçalves [65] for example, used the solubility results of Pollack and Himm [66], to calculate the interaction enthalpy of xenon in a series of $n$-alkanes, concluding that $H_{\text {int }}$ was almost independent of the alkane chain length.

The calculated interaction enthalpies of xenon in hypothetical "all- $\mathrm{CH}_{2}$ " solvents are displayed in Fig. 7 as a function of temperature. The interaction enthalpy for xenon in cycloalkanes (formed exclusively by $\mathrm{CH}_{2}$ units), as that of cyclopentane, can thus be directly compared with that of an "all- $\mathrm{CH}_{2}$ " linear alkane, and is displayed again in Fig. 7. Any differences can be attributed to the singularities introduced by this particular cyclic structure. As can be seen, the experimental interaction enthalpies for the system involving cyclopentane are between $25 \%$ and $62 \%$ more negative than those for the hypothetical "all- $\mathrm{CH}_{2}$ " solvent. This seems to indicate the existence of a particular interaction between xenon and cyclopentane, in agreement with previous studies for other small cycloalkanes.

\section{Conclusions}

The solubility of xenon in cyclopentane has been measured between $254.60 \mathrm{~K}$ and $313.66 \mathrm{~K}$ with an estimated accuracy of $0.3 \%$. The thermodynamic functions of solvation, such as the standard Gibbs energy, enthalpy, entropy and heat capacity of solvation have been calculated from Henry's law coefficients and its temperature dependence for xenon + cyclopentane solutions. The molar enthalpy of solvation was decomposed into its cavity and interaction contributions. The enthalpy of interaction, which in the case of cyclopentane results exclusively from xenon- $\mathrm{CH}_{2}$ interactions, is much more negative than the corresponding enthalpy calculated from (xenon $+n$-pentane) and (xenon $+n$-hexane) solutions. This indicates stronger interactions between xenon and $\mathrm{CH}_{2}$ groups in 
cyclopentane, in agreement with previous studies involving xenon and other small cyclic molecules.

A version of the soft-SAFT theory, developed to describe cyclic molecules, was used to model the experimental results. The theory was able to predict the phase behaviour of pure cyclopentane using molecular parameters of $n$-pentane with any further fitting. Using standard geometric and arithmetic combining rules to obtain unlike mixture parameters, the theory was also able to predict the experimental solubility of xenon in cyclopentane over the range of temperatures studied and the lower solubility of xenon in the cycloalkane with respect to that in $n$-pentane.

\section{Acknowledgements}

EJMF, LFGM and RPMFB acknowledge financial support from the portuguese Fundação para a Ciência e a Tecnologia through project POCTI/QUI/46299/2002 and a PhD grant. MCdR acknowledges the Programme Alßan from European Union Programme of High Level Scholarships for Latin America (identification number E03D21773VE) for a Fellowship. FJB also acknowledges financial support from project number FIS2010-14866 of the Spanish Dirección General de Investigación. Additional support from Universidad de Huelva and Junta de Andalucía is also acknowledged.

\section{References}

[1] J.S. Rowlinson, F.L. Swinton, Liquids and Liquid Mixtures, 3rd ed., Butterworth Scientific, London, 1982

[2] J.C.G. Calado, E.J.M. Filipe, J.N.C. Lopes, Fluid Phase Equilib. 135 (1997) 249.

[3] E.J.M. Filipe, L.A.M. Pereira, L.M.B. Dias, J.C.G. Calado, R.P. Sear, G. Jackson, J. Phys. Chem. B 101 (1997) 11243.

[4] J.C.G. Calado, E.J.M. Filipe, J.N.C. Lopes, J.M.R. Lúcio, J.F. Martins, L.F.G. Martins, J. Phys. Chem. B 101 (1997) 7135.

[5] L.F.G. Martins, E.J.M. Filipe, J.C.G. Calado, J. Phys. Chem. B 105 (2001) 10936.

[6] E.J.M. Filipe, E.J.S. Gomes de Azevedo, L.F.G. Martins, V.A.M. Soares, J.C.G. Calado, C. McCabe, G. Jackson, J. Phys. Chem. B 104 (2000) 1315.

[7] E.J.M. Filipe, L.F.G. Martins, J.C.G. Calado, C. McCabe, G. Jackson, J. Phys. Chem. B $104(2000) 1322$.

[8] V.T. Lam, P. Picker, D. Patterson, Tancréde, J. Chem. Soc., Faraday Trans. II 70 (1974) 1465

[9] PSt.Romain, H.T. Van, D. Patterson, J. Chem. Soc., Faraday Trans. 75 (1979) 1700

[10] St. Romain, P. Van, H.T.D. Patterson, J. Chem. Soc., Faraday Trans. 75 (1979) 1708.

[11] F.J. Blas, L.F. Vega, Mol. Phys. 92 (1997) 135.

[12] F.J. Blas, L.F. Vega, Ind. Eng. Chem. Res. 37 (1998) 660.

[13] J.V. Sengers, R.F. Kayser, C.J. Peters, H.J. White Jr., Equations of State for Fluids and Fluid Mixtures, Elsevier, Amsterdam, 2000.

[14] W.G. Chapman, K.E. Gubbins, G. Jackson, M. Radosz, Fluid Phase Equilib. 52 (1989) 31.

[15] W.G. Chapman, K.E. Gubbins, G. Jackson, M. Radosz, Ind. Eng. Chem. Res. 29 (1990) 1709.

[16] M.S Wertheim, J. Stat. Phys. 35 (1984) 19.

[17] M.S. Wertheim, J. Stat. Phys. 35 (1984) 35.

[18] M.S Wertheim, J. Stat. Phys. 42 (1986) 459

[19] M.S Wertheim, J. Stat. Phys. 42 (1986) 477.

[20] M.S. Wertheim, J. Chem. Phys. 85 (1986) 2929.

[21] M.S. Wertheim, J. Chem. Phys. 87 (1987) 7323.

$22]$ E.A. Müller, K.E. Gubbins, Ind. Eng. Chem. Res. 40 (2001) 2193.

[23] S.P. Tan, H. Adidharma, M. Radosz, Ind. Eng. Chem. Res. 47 (2008) 8063-8082.
[24] Y. Peng, K.D. Goff, M.C. dos Ramos, C. McCabe, Fluid Phase Equilib. 277 (2009) 131.

[25] A. Lymperiadis, C.S. Adjiman, A. Galindo, G. Jackson, J. Chem. Phys. 127 (2007) 234903.

[26] A. Lymperiadis, C.S. Adjiman, G. Jackson, A. Galindo, Fluid Phase Equilib. 274 (2008) 85.

[27] G.L. Pollack, R.P. Kennan, J.F. Himm, P.W. Carr, J. Chem. Phys. 90 (11) (1989) 6569-6579.

28] R.P. Sear, G. Jackson, Mol. Phys. 81 (1994) 801.

[29] I. Mokbel, E. Rauzy, H. Loiseleur, C. Barro, J. Jose, Fluid Phase Equilib. 108 (1995) 103.

[30] R.P. Bonifácio, M.F. Costa Gomes, E.J.M. Filipe, Fluid Phase Equilib. 193 (2002) 41.

[31] J. Carnicer, F. Gibanel, J.S. Urieta, C. Gutierrez Losa, Rev. Acad. Cienc. Zaragoza 34 (1979) 115

[32] J.M. Smith, H.C. Van Ness, M.M. Abbott, Introduction to Chemical Engineering Thermodynamics, 5th ed., McGraw-Hill, New York, 1996.

[33] T.R. Rettich, Y. Paul Handa, R. Battino, E. Wilhelm, J. Phys. Chem. 85 (1981) 3230.

[34] B.B. Benson, D.J. Krause Jr., J. Sol. Chem. 18 (1989) 803.

[35] B.E. Poling, J. Prausnitz, M.J.P. O'Connell, The Properties of Gases and Liquids, 5th ed., McGraw-Hill, New York, 2001, p. 59.

[36] DIPPR data base, AIChE, 1987.

[37] V.G. Baonza, M.C. Alonso, J.N. Delgado, Ber. Bunsen-Ges. Phys. Chem. 96 (1992) 1859.

[38] D.W. Brazier, G.R. Freeman, Can. J. Chem. 47 (1969) 893.

[39] O. Kiyohara, G.C. Benson, A.J. Treszczanowicz, J. Chem. Eng. Data 26 (1981) 263.

[40] J.H. Dymond, J.A. Cholinski, A. Szafranskiand, D. Wyrzykowska-Stankiewicz, Fluid Phase Equilib. 27 (1987) 1.

[41] J.H. Dymond, E.B. Smith, The Virial Coefficients of Pure Gases and Mixtures, Clarendon Press, Oxford, 1980.

[42] E.W. Tiepel, K.E. Gubbins, Can. J. Chem. Eng. 50 (1972) 361-364.

[43] E.W. Tiepel, K.E. Gubbins, J. Phys. Chem. 76 (1972) 3044-3049.

[44] E.C.W. Clarke, D.N. Glew, Trans. Faraday Soc. 62 (1966) 539-545.

[45] M.F. Costa Gomes, J.P. Grolier, Phys. Chem. Chem. Phys. 3 (2001) $1047-$ 1052.

[46] A.M.A. Dias, J.C. Pàmies, J.A.P. Coutinho, I.M. Marrucho, F.F. Vega, J. Phys. Chem. B 108 (2004) 1450.

[47] R.P. Bonifácio, E.J.M. Filipe, C. McCabe, M.F.C. Gomes, A.A.H. Pádua, Mol. Phys. 100 (2002) 2547.

[48] L.M.B. Dias, E.J.M. Filipe, C. McCabe, J.C.G. Calado, J. Phys. Chem. B 108 (2004) 7377.

[49] F.J. Blas, L.F. Vega, J. Chem. Phys. 109 (1998) 7405

[50] J.C. Pàmies, L.F. Vega, Ind. Eng. Chem. Res. 40 (2001) 2532

[51] A. Galindo, P.J. Whitehead, G. Jackson, A.N. Burgess, J. Phys. Chem. 100 (1996) 6781.

[52] S.H. Huang, M. Radosz, Ind. Eng. Chem. Res. 29 (1990) 2284.

[53] S.H. Huang, M. Radosz, Ind. Eng. Chem. Res. 30 (1991) 1994.

[54] G. Jackson, W.G. Chapman, K.E. Gubbins, Mol. Phys. 65 (1988) 1.

[55] W.G. Chapman, G. Jackson, K.E. Gubbins, Mol. Phys. 65 (1988) 1057

[56] J.K. Johnson, J.A. Zollweg, K.E. Gubbins, Mol. Phys. 78 (1993) 591.

[57] J.P. Hansen, I.R. McDonald, Theory of Simple Liquids, 2nd ed., Academic Press, London, 1990.

[58] D. Ghonasgi, M. Llanorestrepo, W.G. Chapman, J. Chem. Phys. 98 (1993) 5662.

[59] R.P.M.F. Bonifácio, L.F.G. Martins, C. McCabe, E.J.M. Filipe, J. Phys. Chem. B (2010).

60] P. Tancréde, P. Bothorel, St. Romain, P.D Patterson, J. Chem. Soc., Faraday Trans. II 73 (1977) 15.

[61] M.B. Ewing, K.N. Marsh, J. Chem. Thermodyn. 9 (1977) 357

[62] St. Romain, P. Van, H.T.D. Patterson, J. Chem. Soc., Faraday Trans. I 75 (1979) 1700.

[63] O. Sinanoglu, Mol. Interact. 3 (1982) 283.

[64] R. Bonifácio, PhD thesis, Instituto Superior Técnico, Lisbon, 2004.

[65] J.J. Moura Ramos, R.M.C. Gonçalves, Thermochim. Acta 190 (1991) 225.

[66] G.L. Pollack, J.F. Himm, J. Chem. Phys. 77 (1982) 3221-3229.

[67] NIST Chemistry Webbook, 1991-2005. Available from: http://webbook.nist.gov/chemistry/.

[68] T.E. Daubert, J. Chem. Eng. Data 41 (1996) 365-372. 